Fecha de recepción: octubre 2019 Fecha de aceptación: diciembre 2019 Versión final: noviembre 2021

\section{Introducción. Interrogantes y respuestas de la Moda en la hipermodernidad}

Patricia M. Doria *

Resumen: En el presente número de Cuadernos del Centro de Estudios en Diseño se analiza la moda, desde sus múltiples aristas desde un complejo y exhaustivo recorrido multidisciplinario. Esta publicación gesta el armado del sustrato vivo, la base y el sostén de un laberinto plural y multidireccional de análisis en el sistema de la moda, el Cuaderno discurre en tres miradas. En primer lugar, una mirada orientada particularmente a las variables entre moda y sociedad, una segunda instancia, donde el eje principal es la moda y la cultura y un tercera mirada, que inserta la innovación y el futuro a través de diversos casos.

Palabras clave: moda - sociedad - innovación - cultura - Diseño - Investigación.

[Resúmenes en inglés y portugués en las páginas 14-15]

${ }^{*}$ ) Doctora en Educación Superior (Universidad de Palermo). Magister en Diseño (Universidad de Palermo). Diseñadora de Indumentaria (Universidad de Buenos Aires). Directora del Área Moda y Tendencias de la Universidad de Palermo. Autora del libro, Vestido de Novia: ritual, símbolo y consumo.

\title{
Introducción
}

La moda es hermana de la muerte, diría Leopardi (2011) y son ambas hijas de la caducidad. König (1972) lo describe de una forma más poética, como el deseo de muerte por mano propia de la moda cuando ella está por alcanzar su cenit.

Simmel (2012) define y ubica a la moda como un fenómeno social que intencionalmente se extiende ilimitadamente. La moda, anuncia su muerte apenas alcanza su objetivo meta, y muere siendo víctima de la contradicción lógica e inherente en sí misma. El cambio y la variabilidad. Según Lipovetsky (1986) la condición efímera de la moda en el periodo hipermoderno es renovarse constantemente, en forma vertiginosa, e instantánea. Este período está caracterizado por el deseo de individualismo y hedonismo.

Bauman (2000) nos indica que la característica más notoria de la hipermodernidad es que sospecha de la certeza y no promete ninguna garantía, no hay una realidad fija, es una realidad atravesada por partes de relatos simultáneos y a su vez totalmente válidos en tiempo y espacios diferentes. 
Cuando analizamos el sistema de la moda en la hipermodernidad, descubrimos como se plantea la recombinación de elementos existentes de un período a otro y de un pensador a otro.

En particular, hablando de esta nueva publicación e investigación se está gestando el armado del sustrato vivo, la base y el sostén de un laberinto plural y multidireccional de análisis en el sistema de la moda.

Este laberinto multidireccional, nos invita a pedernos y a descubrirlo por partes, sin necesidad de ver el todo, sin necesidad de llegar a la salida o resolución del problema.

Si observamos la hipermodernidad esta se encuentra atravesada por el poder individual, la libertad en plural, nadie puede reprochar ni desacreditar a otro, todo es posible. Los discursos son múltiples e inter-dimensionales.

Es el mundo de la libre interpretación, la multiplicidad se presenta en un laberinto, donde la moda intenta dar respuestas a este hombre posmoderno perdido entre avances, retrocesos, caídas en formas circulares, reticulares y sinuosas, donde el verdadero recorrido esta en el viaje y no en llegar; la llegada o la salida no es el objetivo.

En el Hilo de Ariadne como hilo conductor, como posibilidad de salida, Teseo ya no lo precisa, no es importante, -siguiendo la metáfora- no queremos salir del desafío del laberinto, o como nos dice Calabrese (1994) es el encuentro con el monstruo. Ese monstruo amado por diferente, egocéntrico, hedonista. Hoy el exceso pasa a ser la norma de parámetros múltiples e individuales.

Como era también inevitable, en el debate de esta publicación se introdujeron muchos aspectos que nos orientan por los vericuetos de la investigación en moda y probablemente al final de la lectura habremos descubierto si es mejor recorrer los senderos del laberinto, o llegar a la salida. Estos relatos simultáneos segmentados van construyendo las miradas válidas sobre el objeto moda.

\section{Mirada 1. Moda y Sociedad}

Este eje está compuesto por cinco artículos centrados en analizar y teorizar sobre la investigación y la sociedad. Puntualmente el trabajo de M. Lopez Risso, Masculinidad y Moda: el dandismo en la Argentina, describe hitos del Dandismo en la Argentina y su relación entre el mundo de la moda y la construcción de la identidad de la clase dirigente. En sintonía con esta propuesta, el escrito de J. Tidele, Moda y feminismo: la vestimenta como símbolo de protesta, analiza y reflexiona sobre el rol de la moda y el acto de ataviarse como acto político. El aporte de, V. Tuozzo en su escrito, Hipermoda, la moda rizoma, sostiene que la moda a través de su lógica se resignifica, adaptándose a los nuevos paradigmas y a las nuevas cosmovisiones del mundo. En tal sentido el texto denominado La simbología del traje sastre femenino y el discurso de emancipación femenina de Y. Moreira es una indagación sobre el estado del traje sastre femenino como signo en los actuales contextos de la moda. En su escrito titulado La industria Textil y de la Moda, responsabilidad social y la agenda 2030, J. Castro incursiona sobre la industria de la moda, y su responsabilidad en la sociedad. Toda esta interesante reflexión se enmarca en una concepción del proceso proyectual e investigación teórica en sí misma. 


\section{Mirada 2. Moda y Cultura}

Este eje está compuesto por nueve artículos centrados en analizar y teorizar sobre la investigación y cultura, estos conocimientos, e ideas, que atraviesan la enseñanza del diseño de moda con otras artes. En tal sentido el artículo Desfile de Moda: Arte y Perfomance de Sara Peisajovich, asume que el legado modernista invade cada desfile performático y están asociados a partir de los conceptos de hibridación y sus características autorreferenciales. Por su parte el aporte de Lorena Pérez en Observar y consumir moda. Nuevas formas de comunicación digital habilita a comprender el reemplazo de los blogs como medios unipersonales derivando a las redes sociales como una nueva forma de consumir moda. En esta línea Gabriela Gómez del Río, aporta el artículo Los cibergéneros especializados: análisis sobre la modalidad de gestión de contenidos en weblogs independientes de moda. Planteando el interrogante ¿los weblogs son una necesidad del circuito de comunicación digital especializado en moda? Por su parte Patricia Galletti en su escrito Prolijidad y corrección. Vectores de normalización y socialización inter-clase para el cuidado del cliente de élite en una marca comercial porteña de lujo, nos propone una pesquisa de las lógicas de indagación inter-clase y su repercusión en la venta de productos de lujo. Puntualmente con respecto al vestuario los aportes de Cecilia G. García con su mirada sobre la Conservación preventiva de colecciones de vestuario escénico. Colección de vestuario compañía de danza española Ángel Pericet, en su artículo describe y confronta los métodos propuestos de la conservación preventiva con criterios museológicos. Por último y conectado con el anterior artículo, Cecilia Turnes en su escrito, Moda y Vestuario: universos paralelos con infinitas posibilidades de encuentro, plantea un enfoque multidisciplinario entre el complejo mundo del vestuario teatral y del diseño de indumentaria y textil y sus puntos de encuentro en sus lenguajes creativos. Desde el Derecho confluyen tres artículos que analizan la interacción entre moda, publicidad, marketing, y derecho. En la reflexión de Florencia Insausti en su escrito titulado, Moda, Publicidad y Derecho, analiza el universo publicitario y las normativas aplicables para la celebración de contratos y su marco jurídico. Por su parte Pamela Echeverría, en su trabajo Proteger las Creaciones en el Mundo de la Moda, nos muestra las herramientas efectivas para salvaguardar las creaciones y la inversión de capital. En esta línea Constanza Rudi, Emprender en el Mundo de la Moda, analiza la industria de la moda y las posibilidades de nuevos emprendimientos y su protección jurídica.

\section{Mirada 3. Moda Innovación}

Este eje está compuesto por seis artículos y se continúa como curso rector reflexionando acerca de la moda, no obstante el vínculo de análisis se centra en el puente entre moda e innovación, nuevas búsquedas y desafíos. Estos objetos producidos para la moda, abarcan una amplitud que va en sintonía con la disciplina que busca ser teorizada, se enfatiza la variabilidad de los enfoques desde los que se analizan la moda desde una reconfiguración multidisciplinaria. En tal sentido, María Laura Spina en su artículo, El impacto de lenguajes visuales gráficos en Identidad de Indumentaria, asume que la investigación de las tramas gráficas de marcas de moda, son potenciadores de innovación y diferenciadores identita- 
rios, en los actuales mercados competitivos. En sintonía con esta propuesta, Valeria Scalisse, en su escrito Transgresión y Glamour, las portadas de la moda. Un análisis de la pasarela/ vidriera de papel, explica que el objeto moda discurre entre interrogantes sobre íconos de moda, diseño editorial, marcas y la comunicación gráfica de la moda en las revistas de papel. El Fashion Film aparece de la mano del autor Pablo Tesorieri, Fashion Film: tendencia mundial en comunicación, en su trabajo sostiene que el ingreso de la moda al mundo audiovisual, favorece y amalgama el proyecto objeto moda y su correlato como un producto más sensorial que persuasivo que apela a las emociones, algunos autores consideran que el cine se pone al servicio de la moda. Por su parte tanto los aportes de Yanina Moscoso, con su escrito sobre Cosmovisión Textil actual, que enfatiza sobre las posibilidades del material textil, con sus posibilidades de futuro y la preservación del medio ambiente, como el aporte de María Mihanovich con su escrito Slow Fashion en tiempos de redes sociales, implica una nueva forma de consumo más consiente en el mundo de la moda. Por último, Paola Matteazi discurre entre el mundo de la tecnología y el diseño de calzado en su artículo, Tecnología 3D, artesanato y tradición.

\title{
Bibliografía
}

Calabrese, O. (1994). La Era Neobarroca. Madrid: Catedra.

Giacomo, L. (2011). Diálogo entre la Moda y la Muerte. Mexico: Taurus.

König, R. (1972). Sociología de la Moda. Buenos Aires: Carlos Lohle.

Lipovetsky, G. (1986). La era del vacío: Ensayos sobre el Individualismo Contemporáneo. Barcelona: Anagrama.

Lipovetsky, G.(1990). El imperio de lo efímero. La moda y su destino en las sociedades modernas. Barcelona: Anagrama.

Simmel, G. (2012). Filosofía de la moda. México: Casimiro.

Zygmunt, B. (2000). La Modernidad Líquida. México: Fondo de Cultura Económica.

\begin{abstract}
In this issue of the Center for Design Studies, fashion is analyzed, from its multiple edges of a complex and exhaustive multidisciplinary course. This publication breeds the construction of the live substrate, the base and the support of a plural and multidirectional labyrinth of analysis in the fashion system, this writting travel arround three differents points of view. First, a look oriented particularly to the variables between fashion and society, a second instance, where the main focus is fashion and culture and a third look, which inserts innovation and the future through various cases.
\end{abstract}

Keywords: fashion - society - innovation - culture - Design - Research.

Resumo: nesta edição dos Cadernos do Centro de Estudos de Design, a moda é analisada, a partir de suas múltiplas arestas, de um curso multidisciplinar complexo e exaustivo. Esta publicação gera a construção do substrato vivo, a base e o suporte de um labirinto de aná- 
lises plural e multidirecional no sistema de moda, o Notebook é executado em três olhos. Primeiro, um olhar voltado principalmente para as variáveis entre moda e sociedade, uma segunda instância, onde o foco principal é moda e cultura e um terceiro olhar, que insere inovação e futuro em vários casos.

Palavras chave: moda - sociedade - inovação - cultura - design - pesquisa.

[Las traducciones de los abstracts fueron supervisadas por el autor de cada artículo] 